\title{
Feature Cluster: Recent Advances in Exact Methods for Multi-Objective Optimisation
}

\author{
Matthias Ehrgotta,*, Ivana Ljubićb ${ }^{\mathrm{b}}$, Sophie N. Parragh ${ }^{\mathrm{c}}$ \\ ${ }^{a}$ Department of Management Science, Lancaster University Management School \\ Bailrigg, Lancaster LA1 4YX, United Kingdom \\ ${ }^{b}$ ESSEC Business School \\ 3 Av. Bernard Hirsch, B.P. 50105, 95021 Cergy Pontoise Cedex, France \\ ${ }^{c}$ Institute for Transport and Logistics Management, Vienna University of Economics and Business \\ Welthandelsplatz 1, 1020 Vienna, Austria
}

\section{Editorial}

The development of exact methods for multi-objective optimisation is experiencing increasing interest in the OR community as witnessed by the growing body of research emerging from that domain. Recent advances in the development of nonlinear, linear and mixed integer optimisation solvers push the boundaries towards more challenging areas. Hence solving to optimality larger, more difficult (real-world) problems involving several conflicting objectives, has been put within reach. Also, researchers previously working in single objective optimisation are increasingly contributing to the fast progress of the development of exact multi-objective optimisation algorithms. As guest editors we hope that this feature cluster increases the visibility of research concerning exact methods for multi-objective optimisation and to motivate a broader group of researchers to contribute to the field. It presents a collection of the latest research results on exact algorithms for multi-objective optimisation as well as heuristics derived from exact methods.

The call for contributions to the feature cluster solicited 34 submissions, eleven of which were accepted for publication following the stringent review procedures of the European Journal of Operational Research. These papers cover a wide spectrum of topics, from approximation algorithms and complexity theory to representation algorithms, branch and bound, and constraint propagation methods. The authors consider a wide range of problems with multiple objectives: linear, integer and combinatorial optimisation, continuous and (mixed) integer nonlinear optimisation problems all make their appearance, showcasing the breadth and depth of current research in multi-objective optimisation. We briefly summarise the contents of the feature cluster in this editorial.

Andreas Löhne and Benjamin Weißing describe the theoretical background of their open source

\footnotetext{
*Corresponding author, phone number +44 1524593888

Email addresses: m.ehrgott@lancaster.ac.uk (Matthias Ehrgott), ljubic@essec.edu (Ivana Ljubić), sophie.parragh@wu.ac.at (Sophie N. Parragh)
} 
implementation Bensolve of Bensons algorithm and its dual variant. Both algorithms compute primal and dual solutions of linear vector optimisation problems the upper image of which does not contain lines. They pay particular attention to the role of duality for the implementation.

Because the set of nondominated points of multi-objective optimisation problems can be very large or infinite, the issue of representing it by a discrete set satisfying some quality criteria has attracted the interest of researchers over the years. In their contribution, Cristina Bazgan, Florian Jamain and Daniel Vanderpooten introduce the new concept of $\left(\varepsilon, \varepsilon^{\prime}\right)$-kernels $(\varepsilon$-kernels when $\varepsilon^{\prime}=\varepsilon$ is possible). They first establish some general properties of $\varepsilon$-kernels and then propose generic algorithms computing in polynomial time $\varepsilon$-kernels for the bi-objective case. For more than two objectives, they prove some existence results.

Referring to the same topic, Lizhen Shao and Matthias Ehrgott address the problem of representing the continuous but nonconvex set of nondominated points of a multi-objective linear optimisation problem by a finite subset of such points. They prove that a related decision problem is NP-complete. They illustrate the drawbacks of known methods and propose a new algorithm that overcomes the limitations, but preserve the advantages of the existing methods, proving that their algorithm computes a set of evenly distributed nondominated points with quality guarantees.

Closely related to finding representations of nondominated sets is the subject of approximation algorithms for multi-objective optimisation problems. Christina Büsing, Kai-Simon Goetzmann, Jannik Matuschke and Sebastian Stiller investigate relationships between reference point algorithms, i.e., algorithms that find a solution closest to a given reference point in the objective space, and approximation algorithms for multi-objective discrete optimisation problems. They establish that, in terms of computational complexity, computing approximate reference point solutions is polynomially equivalent to approximating the Pareto set. They also show how a number of general algorithmic techniques in single objective optimisation can be lifted to reference point optimisation.

Multi-objective optimisation procedures usually proceed by iteratively producing new solutions. For this purpose, a key issue is to determine and efficiently update the search region, which corresponds to the part of the objective space where new nondominated points could lie. Kerstin Dächert, Kathrin Klamroth, Renaud Lacour and Daniel Vanderpooten elaborate a specific neighborhood structure among local upper bounds thanks to which the update of the search region with respect to a new point can be performed more efficiently compared to existing approaches. Moreover, the neighborhood structure provides new insights into the search region and the location of nondominated points.

The just mentioned fifth paper provides the link to the following sequence of six papers that address more specific algorithmic issues.

Branch and bound is a well known generic method for computing an optimal solution of a 
single objective optimisation problem. Anthony Przybylski and Xavier Gandibleux survey the literature on multi-objective branch and bound algorithms covering concepts, components (such as bound sets) and published algorithms. Their review focuses on contributions belonging to the class of linear optimisation problems with binary variables or mixed binary and continuous variables. They propose a unified architecture of multi-objective branch and bound methods and review published algorithms which compute the set of all nondominated points.

Multi-objective integer optimisation problems are also the topic of the contribution by Natashia Boland, Hadi Charkhgard and Martin Savelsbergh. Their quadrant shrinking method is a simple and efficient algorithm for solving integer optimisation problems with three objectives. It finds all nondominated points of a tri-objective integer optimisation problem. The algorithm solves at most $3\left|\mathcal{Y}_{N}\right|+1$ single objective integer optimisation problems when computing the set of nondominated points, where $\mathcal{Y}_{N}$ is the set of all nondominated points.

Elena Fernández, Miguel A. Pozo, Justo Puerto and Andrea Scozzari consider a specific nonlinear multi-objective combinatorial optimisation problem, namely the multi-objective spanning tree problem, where the ordered median objective function is used as an averaging operator to aggregate the vector of objective values of feasible solutions. Different mixed integer linear optimisation models are proposed, based on the most relevant spanning tree models in the literature. These formulations are analysed and several enhancements presented.

In their second contribution to this issue, Natashia Boland, Hadi Charkhgard and Martin Savelsbergh present a new algorithm for optimising a linear function over the set of efficient solutions of a multi-objective integer optimisation problem. The success of the algorithm relies on the efficiency of a new algorithm for enumerating the nondominated points of a multi-objective integer optimisation problem. It employs a novel criterion space decomposition scheme which limits the number of subspaces that are created, and limits the number of sets of disjunctive constraints required to define the single-objective integer optimisation problem that searches a subspace for a nondominated point.

The paper by Valentina Cacchiani and Claudia D'Ambrosio proposes a heuristic algorithm for multi-objective convex mixed integer nonlinear optimisation problems that is based on branch and bound. The heuristic computes a good approximated set of nondominated points of such problems. It starts with a set of feasible points, obtained, at the root node of the enumeration tree, by iteratively solving, with an $\varepsilon$-constraint method. Lower bounds are derived by optimally solving nonlinear optimisation problems. Each leaf node of the enumeration tree corresponds to a convex multi-objective nonlinear optimisation problem, which is solved heuristically by varying the weights in a weighted sum approach. In order to improve the obtained points and remove dominated ones, a tailored refinement procedure is designed.

The feature cluster concludes with a contribution by Benjamin Martin, Alexandre Goldsztejn, 
Laurent Granvilliers and Christophe Jermann on constraint propagation using dominance in interval branch and bound for nonlinear bi-objective optimisation. They argue that although constraint propagation has been widely used in nonlinear single objective optimisation inside interval branch and bound algorithms as an efficient way to discard infeasible and nonoptimal regions of the search space, constraint propagation is not commonly used when considering bi-objective optimisation. The difficulty is in the exploitation of dominance relations in order to discard the non-Pareto optimal solutions inside a decision domain. Their interval branch and bound algorithm integrates dominance contractors and constraint propagation mechanisms that exploit an upper bound set using dominance relations.

\section{Acknowledgments}

The editors are grateful to the 42 colleagues who acted as reviewers for the submitted papers for their contribution in ensuring the quality of this feature cluster. Without their hard work it would not exist. 